\title{
Effect of active smoking on the human bronchial epithelium
} transcriptome

\author{
Raj Chari*1, Kim M Lonergan ${ }^{1}$, Raymond T Ng${ }^{2}$, Calum MacAulay ${ }^{3}$, \\ Wan L Lam ${ }^{\dagger 1}$ and Stephen Lam ${ }^{\dagger 3}$
}

Address: ${ }^{1}$ Department of Cancer Genetics and Developmental Biology, British Columbia Cancer Research Centre, Vancouver, BC, Canada, 2Department of Computer Science, University of British Columbia, Vancouver, BC, Canada and ${ }^{3}$ Department of Cancer Imaging, British Columbia Cancer Research Centre, Vancouver, BC, Canada

Email: Raj Chari* - rchari@bccrc.ca; Kim M Lonergan - klonergan@bccrc.ca; Raymond T Ng - rng@cs.ubc.ca; Calum MacAulay - cmacaula@bccrc.ca; Wan L Lam - wanlam@bccrc.ca; Stephen Lam - slam@bccancer.bc.ca

* Corresponding author †Equal contributors

Published: 29 August 2007

BMC Genomics 2007, 8:297 doi:10.1/86/147|-2/64-8-297
Received: 2 January 2007

Accepted: 29 August 2007

This article is available from: http://www.biomedcentral.com/I47I-2I64/8/297

(C) 2007 Chari et al; licensee BioMed Central Ltd.

This is an Open Access article distributed under the terms of the Creative Commons Attribution License (http://creativecommons.org/licenses/by/2.0), which permits unrestricted use, distribution, and reproduction in any medium, provided the original work is properly cited.

\begin{abstract}
Background: Lung cancer is the most common cause of cancer-related deaths. Tobacco smoke exposure is the strongest aetiological factor associated with lung cancer. In this study, using serial analysis of gene expression (SAGE), we comprehensively examined the effect of active smoking by comparing the transcriptomes of clinical specimens obtained from current, former and never smokers, and identified genes showing both reversible and irreversible expression changes upon smoking cessation.
\end{abstract}

Results: Twenty-four SAGE profiles of the bronchial epithelium of eight current, twelve former and four never smokers were generated and analyzed. In total, 3, I I I,47I SAGE tags representing over 110 thousand potentially unique transcripts were generated, comprising the largest human SAGE study to date. We identified 1,733 constitutively expressed genes in current, former and never smoker transcriptomes. We have also identified both reversible and irreversible gene expression changes upon cessation of smoking; reversible changes were frequently associated with either xenobiotic metabolism, nucleotide metabolism or mucus secretion. Increased expression of TFF3, CABYR, and ENTPD8 were found to be reversible upon smoking cessation. Expression of GSK3B, which regulates COX2 expression, was irreversibly decreased. MUC5AC expression was only partially reversed. Validation of select genes was performed using quantitative RT-PCR on a secondary cohort of nine current smokers, seven former smokers and six never smokers.

Conclusion: Expression levels of some of the genes related to tobacco smoking return to levels similar to never smokers upon cessation of smoking, while expression of others appears to be permanently altered despite prolonged smoking cessation. These irreversible changes may account for the persistent lung cancer risk despite smoking cessation.

\section{Background}

Lung cancer has the highest mortality rate among all types of malignancies, accounting for approximately $29 \%$ of all cancer-related deaths in the United States [1]. It has been estimated that in 2006 alone, the number of new lung cancer cases will exceed 174,000 and approximately 
163,000 people will die of this disease [1]. Tobacco smoking accounts for $85 \%$ of the lung cancers. Former heavy smokers remain at an elevated risk for developing lung cancer even years after they stop smoking [2,3]. Fifty percent of newly diagnosed lung cancer patients are former smokers [4]. It is therefore important to understand the effects of tobacco smoking on the bronchial epithelium in both active and former smokers.

Recently, a large-scale microarray study characterized gene expression differences between current, former, and never smokers [5], and identified specific genes related to xenobiotic functions, anti-oxidation, cell adhesion and electron transport to be more highly expressed in current smokers relative to never smokers. Genetic regulators of inflammation and putative tumor suppressor genes exhibited decreased expression in current smokers relative to never smokers. Most significantly, a number of genes were identified that exhibited irreversible expression changes upon smoking cessation.

Additional reports have also identified increased expression of various xenobiotic metabolic enzymes including members of the cytochrome P450 (CYP) and glutathione S-transferase (GST) families of proteins in response to cigarette smoke exposure [5-10]. CYP enzymes mediate the conversion of benzo (a) pyrene and other polycyclic aromatic hydrocarbons (PAH) to carcinogenic intermediates that interact with genomic DNA [8], thus contributing to the formation of DNA adducts in smokers [11-13]. Members from both of the CYP and GST gene families have been implicated as potential susceptibility loci mediated by the presence of single nucleotide polymorphisms (SNPs) leading to aberrant expression in response to smoking $[14,15]$.

Another important process associated with tobacco smoke exposure is the airway mucosal response. In animal models, it has been shown that exposure to cigarette smoke induces goblet cell hyperplasia with accompanied mucus production [16,17]. Moreover, mucin 5 (MUC5AC), has been shown to be the most highly expressed mucin in bronchial secretions [18], induced in response to cigarette smoke through an EGFR-dependent mechanism [19]. However, beyond this, little is known of the genes that are associated with airway remodeling as a result of tobacco smoking.

Serial analysis of gene expression (SAGE) is a quantitative experimental procedure widely used to determine expression profiles through the enumeration of short sequence tags and their relative abundance [20]. Although the construction and sequencing of an individual SAGE library is expensive and laborious compared to microarray analysis, SAGE offers the invaluable potential for gene discovery as the analysis is not limited to genes represented on an array. Moreover, comparisons between independent experiments can be performed without sophisticated normalization $[21,22]$.

In this study, we compare the bronchial epithelial transcriptomes of current, former, and never smokers to determine the effect of active smoking on gene expression using bronchial brushings from the peripheral sub-segmental airways. Genes whose expression is reversible upon smoking cessation are expected to differ in abundance between current and former smokers, but are similar between former and never smokers. Conversely, gene expression that is irreversible upon smoking cessation will show similar levels in current and former (ever) smokers but differ between ever and never smokers. Here, we focus on identifying both reversible and irreversible gene expression changes and specifically consider these expression changes in the context of airway mucosal response, and susceptibility to cancer development.

\section{Results and Discussion SAGE library statistics}

Twenty-four SAGE libraries were constructed from bronchial epithelial specimens acquired from eight current smokers, twelve former smokers and four never smokers (Table 1). A former smoker was defined as someone who had stopped smoking for one year or longer. The smoking status was verified using exhaled carbon monoxide monitoring. Raw SAGE data for these transcriptomes has been made publicly available at National Center for Biotechnology Information (NCBI) Gene Expression Omnibus (GEO) with series accession number GSE5473. From these 24 libraries, we have collectively sequenced $3,111,471$ SAGE tags, yielding 231,866 unique tags, making this the largest human SAGE study reported to date (Figure 1A). Of the unique tags, nearly half were present in more than one library at a tag count of one or greater, and $70 \%(82,983$ tags $)$ of these tags map to a UniGene cluster. As multiple tags frequently map to the same UniGene cluster, 25,653 unique UniGene clusters are represented in our dataset. Significantly, over 27,000 tags did not map to existing annotated genes, reiterating the continuing potential of re-mining this large dataset as tag-togene mapping improves with the continuing annotation of human transcripts.

\section{Analysis of the current, former and never smoker transcriptomes}

We determined both the number of SAGE tags present in each of the current, former and never smoker transcriptomes, as well as those tags equally represented among the three different datasets. The criteria chosen for preferential expression was a threshold of a raw tag count of $\geq 2$ across all samples in a particular set, but not existing in the other 
Table I: Demographics of subjects in study

\begin{tabular}{|c|c|c|c|c|c|c|c|}
\hline Sample Name & Gender & $\begin{array}{l}\text { Age at } \\
\text { Analysis }\end{array}$ & Pack-Years & $\begin{array}{l}\text { Smoking } \\
\text { Status* }\end{array}$ & $\begin{array}{l}\text { Years of smoking } \\
\text { cessation (years) }\end{array}$ & $\begin{array}{c}\text { Lung function } \\
\text { (predicted FEV }, \%)\end{array}$ & $\begin{array}{l}\text { Name from } \\
\text { previous study** }\end{array}$ \\
\hline Current I & $\mathrm{F}$ & 63 & 40 & CS & $N / A$ & 69 & $B E-13$ \\
\hline Current 2 & $M$ & 56 & 62 & CS & $N / A$ & 89 & BE-7 \\
\hline Current 3 & $\mathrm{~F}$ & 63 & 44 & CS & $N / A$ & 96 & $B E-12$ \\
\hline Current 4 & $M$ & 68 & 81 & CS & $\mathrm{N} / \mathrm{A}$ & 76 & BE-I \\
\hline Current 5 & $M$ & 64 & 45 & CS & $N / A$ & 73 & $\mathrm{BE}-2$ \\
\hline Current 6 & $M$ & 66 & 53 & CS & $\mathrm{N} / \mathrm{A}$ & 85 & - \\
\hline Current 7 & $M$ & 52 & 48.1 & CS & $\mathrm{N} / \mathrm{A}$ & 63 & - \\
\hline Current 8 & $\mathrm{~F}$ & 55 & 34.4 & CS & $N / A$ & 81 & - \\
\hline Former I & $M$ & 68 & 33 & FS & 19 & 50 & BE-3 \\
\hline Former 2 & $M$ & 69 & 100 & FS & 1 & 21 & $B E-4 A / 4 B$ \\
\hline Former 3 & $M$ & 68 & 30 & FS & I & 30 & BE-9 \\
\hline Former 4 & M & 70 & 75 & FS & 17 & 76 & BE-5 \\
\hline Former 5 & $M$ & 67 & 55 & FS & 5 & $N / A$ & BE-6 \\
\hline Former 6 & $M$ & 65 & 82 & FS & 10 & 59 & BE- 10 \\
\hline Former 7 & $\mathrm{~F}$ & 56 & 64 & FS & 1.5 & 71 & BE-IIA \\
\hline Former 8 & $\mathrm{~F}$ & 63 & 45 & FS & 4.5 & 83 & BE-I4 \\
\hline Former 9 & $M$ & 72 & 40 & FS & 32 & 87 & BE-I5 \\
\hline Former 10 & $\mathrm{~F}$ & 71 & 56 & FS & 16 & 58 & BE- 16 \\
\hline Former II & $M$ & 72 & 63 & FS & 6 & $N / A$ & BE-8B \\
\hline Former 12 & $M$ & 69 & 55.3 & FS & 21 & 57 & - \\
\hline Never I & $M$ & 58 & 0 & NS & $N / A$ & 115 & - \\
\hline Never 2 & $\mathrm{~F}$ & 56 & 0 & NS & N/A & 104 & - \\
\hline Never 3 & $M$ & 53 & 0 & NS & $N / A$ & N/A & - \\
\hline Never 4 & $\mathrm{~F}$ & 81 & 0 & NS & $N / A$ & N/A & - \\
\hline
\end{tabular}

* CS = Current Smoker, FS = Former Smoker, NS = Never Smoker

**Subset of samples were used in a previous study by Lonergan et al 2006 [55]

sets. Out of 3,033 tags expressed in all current smokers, we found 227 preferentially expressed tags (Additional file $1)$. In former smokers, 102 tags were found to be preferentially expressed (out of 2,579 tags) (Additional file 2), and in never smokers, 2,013 tags were found to be preferentially expressed (out of 5,192) (Additional file 3). It should be noted that the number of tags preferential to the never smoker set is substantially higher, most likely due to the lower sample size of never smokers relative to the other two groups. However, since we are using never smokers as a reference, a larger transcriptome will lessen the likelihood that we would find transcripts that are preferentially expressed in current and former smokers that were not correct. Looking at those tags which are common to all three groups, it was found that 1,970 tags (mapping to 1,733 unique genes) were expressed in all 24 libraries (Additional file 4). A Venn diagram illustrating the expression patterns of these three groups is given in Figure 1B.

\section{Genes differentially expressed between current and never smokers}

We used a Mann Whitney U test to identify tags differentially expressed in the transcriptomes of current and never smokers. Using cut-off requirements of $p \leq 0.05$, and a fold change of the means $\geq 2$, we identified 609 SAGE tags (mapping to 487 unique genes) to be differentially expressed between current and never smokers (Additional file 5).

\section{Supervised clustering and principal component analysis (PCA) of current, former and never smokers}

Using the 609 tags found to be differentially expressed between current and never smokers (Additional file 5), single link hierarchical clustering was performed using the program Genesis [23]. We hypothesized that these 609 tags would classify current, former and never smokers. Indeed, distinct clusters emerged separating groups of current and former smokers with one exception of Current 4 (Figure 2A). Of note, the former smoker who ceased smoking for only one year (Former 2) clustered with other former smokers. Moreover, principal component analysis (PCA) further validates the distinct groups of current, former and never smokers (Figure 2B).

\section{Reversible gene expression changes upon cessation of smoking}

To determine reversibility of smoking-related gene expression changes, we intersected tags differentially expressed between current and never smokers against tags showing significant expression difference between current and former smokers using similar criteria. By comparing these two sets, we can deduce which gene expressions are revers- 
A

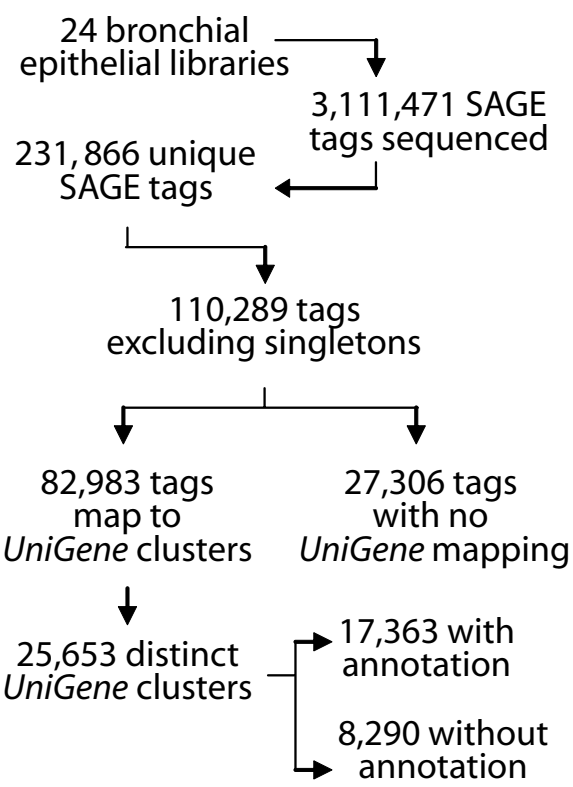

B

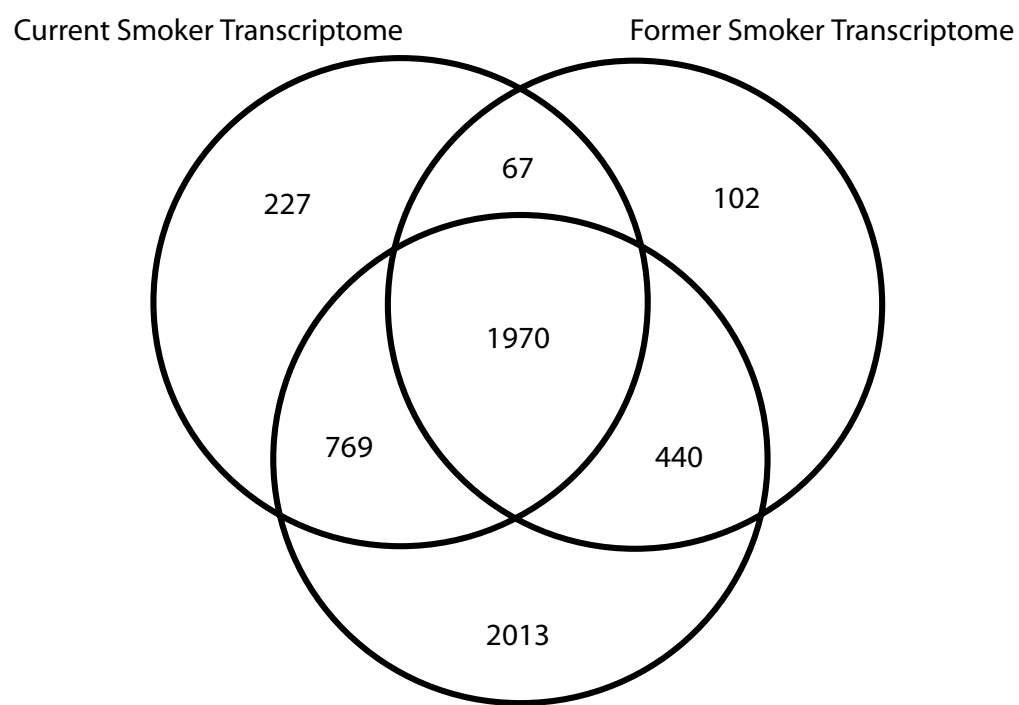

Never Smoker Transcriptome

\section{Figure I}

(A) SAGE library statistics: Summary statistics of the 24 SAGE libraries analyzed in this study. Mapping information was based on the May I0th, 2006 version of SAGEGenie [45]. In total, over 3,000,000 SAGE tags were sequenced, with over I I0,000 unique tags represented upon the exclusion of super singleton tags. (Super singleton tags are tags which have a count of $I$ in a single library only). Approximately $75 \%$ of these I I0,000 unique tags, (potentially representing as many unique transcripts), mapped to an annotated UniGene cluster. As multiple SAGE tags frequently map to the same UniGene cluster, we have identified at a total of 25,653 distinct UniGene clusters within our dataset, approximately $68 \%$ of which represent previously characterized genes. Notably, $25 \%$ of the unique tags had no mapping, suggesting much information is currently unknown. (B) Transcriptome Venn diagram: Venn diagram of the transcriptomes of current, former and never smokers. Reported is the number of tags which are expressed in every library group at a raw tag count greater than or equal to 2, representing the tags which are constitutively expressed in each set. Nearly 2000 SAGE tags, mapping to over 1700 genes are common to all 24 SAGE libraries. A lower number of never smokers may have contributed to a higher number of preferentially expressed transcripts in this group.

ible, i.e., which genes are largely influenced by active smoking. This analysis yielded 161 tags mapping to 121 unique genes, which were deemed statistically significant, and representing $26 \%$ of the total number of differentially expressed tags between current and never smokers (Figure $3 \mathrm{~A}$, Additional file 6). Further analysis of these 121 differentially expressed genes has identified two main functions: xenobiotic metabolism and nucleotide metabolism (representing 33\% of the reversible gene expression changes) (Table 2) and airway mucus secretion (representing $12 \%$ of the reversible gene expression changes) (Table 3). Genes related to oxidative stress were considered as part of the xenobiotic metabolism/nucleic acid metabolism category, and those genes previously associated with xenobiotic metabolism and oxidative stress through smoke exposure were among those identified $[5,24,25]$.
For example, ectonucleoside triphosphate diphosphohydrolase 8 (ENTPD8), an extracellular nucleic acid metabolic enzyme, is among 18 novel genes (labeled in bold in Table 2) not previously associated with smoking and whose expression is increased in response to active smoking. According to enzyme classification, ENTPD8 is involved in purine and pyrimidine metabolism. Hence, this gene may potentially play a role in the chemical formation of DNA adducts.

Gene expression related to airway muco-ciliary function is also elevated in both current versus former smokers and current versus never smokers (Table 3). For example, trefoil factor 3 (TFF3), a structural component of mucus that is elevated in inflammatory response $[26,27]$, and calcium binding tyrosine- $(Y)$ phosphorylation regulated (CABYR), originally shown to be localized in the principal part of 


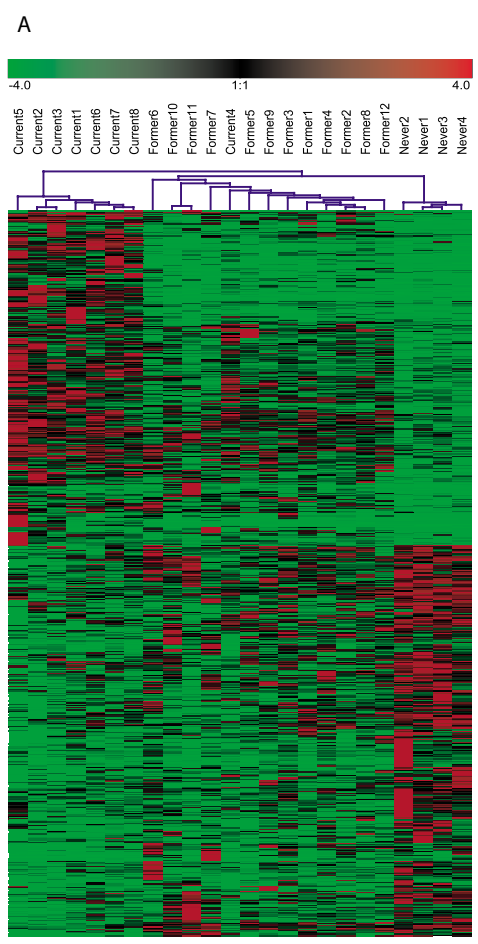

B

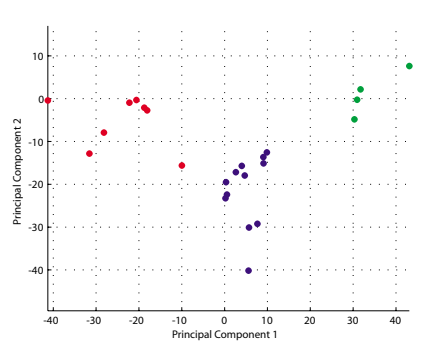

\section{Figure 2}

(A) Cluster analysis of current, former and never smokers: Single link hierarchical clustering using the 609 SAGE tags comprised in Additional file 5 representing tags differentially expressed between current and never smokers. Distance measure used was a Euclidean distance. The visualization package Genesis [23] was used for clustering. Green rectangles represent samples with lower expression for the particular gene amongst the samples, and red rectangles represent samples where the gene is highly expressed relative to other samples. (B) Principal component analysis of current, former and never smokers. Expression values used were scaled to tags per million (TPM). Each tag was then normalized by dividing its value by the maximum value for that tag seen in all the libraries. Subsequently, this value was then multiplied by 6 and then subtracted by 3 to put the values ratios in the range of -3 to 3. A co-variance based approach was used and the statistics toolbox in MatLab (Mathworks) was used. Current smokers are represented in red, former smokers are represented in blue and never smokers are represented in green. the human sperm flagellum [28], are both highly expressed in current smokers relative to former and never smokers. Though TFF3 was recently shown to be expressed in response to chronic exposure of nicotine in intestinal cells [29], this is the first report of this gene being overexpressed within the bronchial epithelium in response to active smoking. Based on its assumed role in sperm motility, CABYR may be involved in ciliary function associated with muco-ciliary clearance response within the lung [28]. Interestingly, overexpression of $C A B Y R$ variants have been reported in a variety of brain tumors [30], suggesting a role in carcinogenesis. Previous observation of increased MUC5AC expression in current relative to never smokers and increased expression of microseminoprotein, beta- (MSMB), a gene shown to be present in mucosal secretions [31], supports the possibility of induction of airway mucosal response in active smokers [5,24,25,32].

\section{Irreversible gene expression changes upon cessation of smoking}

By intersecting genes which are differentially expressed between current and never smokers with those that are different between former and never smokers, we can identify irreversible gene expression changes upon smoking cessation. This analysis yielded 152 tags (124 unique genes) meeting the criteria of statistical significance ( $p \leq$ 0.05 ) at a fold change $\geq 2$ (Figure 3B, Additional file 7). Although genes identified by this analysis appear to be functionally diverse, a small number of genes related to the cell cycle process and DNA repair have been identified here. For example, expression of $P 21 / C d c 42 /$ Rac1-activated kinase 1 (PAK1), cyclin D1 (CCND1), and cyclin G2 (CCNG2) all appear to be irreversibly lower in ever (former and current) smokers relative to never smokers. This finding is consistent with a previous report of increased inhibition of cell proliferation through genes such as CDKN1A in a higher stage (GOLD-2) of chronic obstructive pulmonary disease (COPD) versus the lowest stage (GOLD-0) [33].

We also found genes associated with DNA repair to be differentially expressed between current and never smokers, but similar between current and former smokers. APEX nuclease (multifunctional DNA repair enzyme) 1 (APEX1), High-mobility group box 1 (HMGB1), REV1-like (REV1L), and Tumor suppressor candidate 4 (TUSC4) are repair genes which we have found to be irreversibly under-expressed in ever smokers. Significantly, APEX1 has been shown to harbor SNPs associated with lung cancer susceptibility [34]. Moreover, REV1L is involved with the recruitment of DNA polymerase eta to assist in DNA replication at arrested replication forks in areas of DNA lesions such as those formed by thymine dimmers [35,36]. TUSC4, also known as NPRL2, has recently been shown to increase 
Table 2: Reversible gene expression upon smoking cessation related to xenobiotic metabolism and DNA adduct formation (genes in bold have not been previously associated with smoking)

\begin{tabular}{|c|c|c|c|c|c|c|}
\hline Tag & Gene Symbol & Gene Name & CS Mean* & FS Mean* & NS Mean* & P-Value (CVsF) \\
\hline GGCCCAGGCC & ALDH3AI & Aldehyde dehydrogenase 3 family, memberAI & 4355 & 313 & 261 & 0.00002 \\
\hline TTAAAAATTC & $\mathrm{ADH7}$ & Alcohol dehydrogenase 7 (class IV) & 899 & 145 & 130 & 0.00002 \\
\hline AGGTCTGCCA*** & AKRIC2 & Aldo-keto reductase family 1 , member $\mathrm{C} 2$ & 547 & 116 & 74 & 0.00002 \\
\hline AATGCTTTTA & CYPIBI & Cytochrome P450, family I, subfamily B, polypeptide I & 204 & 13 & 0 & 0.00002 \\
\hline TTGGAATCCA & STAU2 & Staufen, RNA binding protein, homolog 2 (Drosophila) & 67 & 17 & 23 & 0.00002 \\
\hline TTATCAAATC & NQOI & $\mathrm{NAD}(\mathrm{P}) \mathrm{H}$ dehydrogenase, quinone $\mathrm{I}$ & 809 & 202 & 149 & 0.00003 \\
\hline CAAATAAACC & PIR & Pirin (iron-binding nuclear protein) & 260 & 47 & 43 & 0.00003 \\
\hline GGCCCCATTT & CBRI & Carbonyl reductase I & 144 & 31 & 24 & 0.00003 \\
\hline TATTTTTGTT & TXNRDI & Thioredoxin reductase I & 250 & 88 & 78 & 0.00006 \\
\hline GGTGGTGTCT & GPX2 & Glutathione peroxidase 2 (gastrointestinal) & 384 & 40 & 46 & 0.00011 \\
\hline TATTTTTGAA & DRB I & Developmentally regulated RNA-binding protein I & 204 & 32 & 22 & 0.00011 \\
\hline TGGGAGTGGG** & NMNAT2 & Nicotinamide nucleotide adenylyltransferase 2 & 175 & 17 & 9 & 0.00011 \\
\hline CAAGACCAGT & GSTA2 & Glutathione S-transferase A2 & 1436 & 485 & 528 & 0.00019 \\
\hline GCTTGAATAA & AKRIBIO & Aldo-keto reductase family I, member BI0 (aldose reductase) & 332 & 10 & 15 & 0.0003 \\
\hline GTGCAGGGAG & SPDEF & SAM pointed domain containing ets transcription factor & 239 & 64 & 50 & 0.0003 \\
\hline GGAGGCTTCC & MECR & Mitochondrial trans-2-enoyl-CoA reductase & 85 & 26 & 22 & 0.0003 \\
\hline GTGATGTAAG & SRXNI & Sulfiredoxin I homolog (S. cerevisiae) & 63 & 14 & 11 & 0.0003 \\
\hline TATGCTTTAA & NT5DCI & 5'-nucleotidase domain containing I & 59 & 23 & 24 & 0.0003 \\
\hline GAACGCCTAA & DPYSL2 & Dihydropyrimidinase-like 2 & $\mathbf{I}$ & 21 & 23 & 0.0004 \\
\hline TTTTCTGAAA & TXN & Thioredoxin & 698 & 326 & 212 & 0.00048 \\
\hline CTTGCATAAG & CYPIAI & Cytochrome P450, family I, subfamily A, polypeptide I & 89 & 2 & 0 & 0.00048 \\
\hline GCAAGAAGAG & ALDH3AI & Aldehyde dehydrogenase 3 family, memberAI & 77 & 10 & 2 & 0.00048 \\
\hline AGAACAAAAC & PRDXI & Peroxiredoxin I & 1043 & 418 & 510 & 0.00071 \\
\hline AAATATTTAA & SLC35A3 & Solute carrier family 35 , member $A 3$ & 47 & 14 & 19 & 0.00071 \\
\hline CGGCTGAATT & PGD & Phosphogluconate dehydrogenase & 252 & 104 & 80 & 0.00106 \\
\hline CTTATCAGTA & BTBD7 & BTB (POZ) domain containing 7 & 94 & 22 & 2 & 0.00106 \\
\hline AAGAGTTTTG & AKRIBI & Aldo-keto reductase family I, member $\mathrm{BI}$ (aldose reductase) & 25 & 5 & 6 & 0.00141 \\
\hline GCTGAGATGA** & CYP4FII & Cytochrome P450, family 4, subfamily F, polypeptide II & 22 & 6 & 2 & 0.00141 \\
\hline GGCGCCTCCT & TALDOI & Transaldolase I & 232 & 76 & 94 & 0.00152 \\
\hline GACACAGCAA & ENTPD8 & Ectonucleoside triphosphate diphosphohydrolase 8 & 24 & 3 & 2 & 0.00159 \\
\hline CAGTCTAAAA & UCHLI & $\begin{array}{c}\text { Ubiquitin carboxyl-terminal esterase LI (ubiquitin } \\
\text { thiolesterase) }\end{array}$ & 92 & 4 & 0 & 0.00197 \\
\hline ACATCCTAGG & ALDHIAI & Aldehyde dehydrogenase I family, member AI & 60 & 28 & 30 & 0.00216 \\
\hline TTAGAAGGAA & NQOI & $\mathrm{NAD}(\mathrm{P}) \mathrm{H}$ dehydrogenase, quinone I & 41 & 14 & 9 & 0.00216 \\
\hline AGGTCTACCA & AKRIC2 & Aldo-keto reductase family 1 , member $\mathrm{C} 2$ & 270 & 32 & 8 & 0.00292 \\
\hline ATTAGGCCTG & TXNRDI & Thioredoxin reductase I & 51 & 19 & 17 & 0.00297 \\
\hline GAGAGCTTTG & AKRIC3 & Aldo-keto reductase family $\mathrm{I}$, member $\mathrm{C} 3$ & 149 & 21 & 22 & 0.00298 \\
\hline TACGCTTGGT & CYB5RI & Cytochrome b5 reductase I & 68 & 32 & 30 & 0.00298 \\
\hline CACTGCCTTG & FTHI & Ferritin, heavy polypeptide I & 59 & 23 & 17 & 0.00298 \\
\hline CTGCTGCACT & GSR & Glutathione reductase & 126 & 54 & 50 & 0.0041 \\
\hline GGCAAAATTA & SLC35A3 & Solute carrier family 35 , member $A 3$ & 73 & 32 & 35 & 0.0041 \\
\hline ACCTTGGGGT & NQOI & $\mathrm{NAD}(\mathrm{P}) \mathrm{H}$ dehydrogenase, quinone I & 73 & 19 & 6 & 0.0041 \\
\hline AATGTTCAGG & COQ6 & Coenzyme Q6 homolog, monooxygenase (yeast) & 29 & 12 & 4 & 0.00708 \\
\hline CACTGACCAG & NOD27 & Nucleotide-binding oligomerization domains 27 & 31 & 10 & 0 & 0.00927 \\
\hline CTCGGAGGCC & SEPXI & Selenoprotein $\mathbf{X}, \mathbf{I}$ & 71 & 32 & 28 & 0.00956 \\
\hline CTCCAAAAAA & CPSF2 & Cleavage and polyadenylation specific factor $2,100 \mathrm{kDa}$ & 118 & 44 & 14 & 0.02013 \\
\hline AATGGAAACT & GCLM & Glutamate-cysteine ligase, modifier subunit & 34 & 16 & 9 & 0.03186 \\
\hline
\end{tabular}

*Mean in tags per million (TPM)

** Changed mapping with TAGMapper [56]

sensitivity to cisplatin [37]. Finally, HMGB1 has also been suggested to be involved with the recruitment of other repair-related proteins [38].

It should be noted that a significant proportion of former smokers in our sample set exhibited low $\mathrm{FEV}_{1}$ levels, raising the possibility that airflow obstruction may be a confounding issue in this analysis. To address this, we used the 20 individuals with available $\mathrm{FEV}_{1}$ data to compare individuals with moderate or severe COPD $\left(\mathrm{FEV}_{1}<80 \%\right.$, $\mathrm{n}=12$ ) with those individuals that would be classified with at most mild COPD $\left(\mathrm{FEV}_{1} \geq 80 \%, \mathrm{n}=8\right)$ according to the GOLD staging classification based on $\mathrm{FEV}_{1}$ status $[39,40]$. Of the 157 tags differentially expressed between these two groups, only 6 tags overlap with our list of irreversible genes (Additional file 8 ). This minimal overlap suggests that the irreversible genes identified are not significantly associated with airway obstruction based on $\mathrm{FEV}_{1}$ status. Nonetheless, airway obstruction should be considered in the interpretation of differential gene expression between current and former smokers. 
A

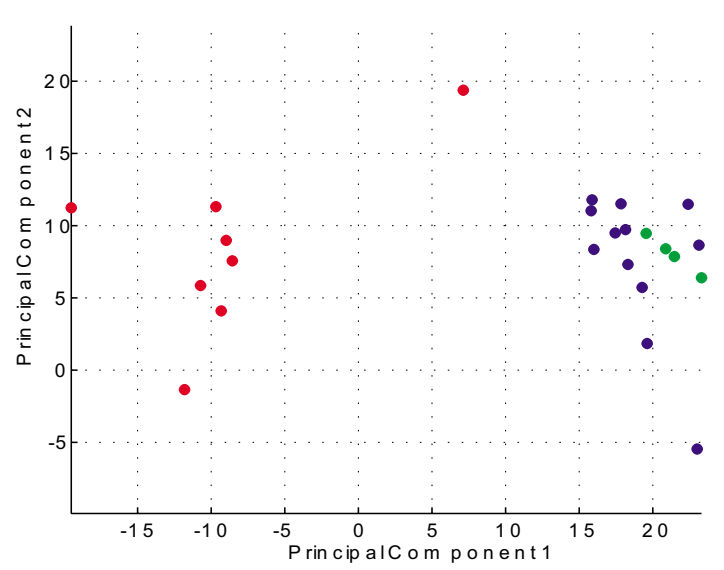

B

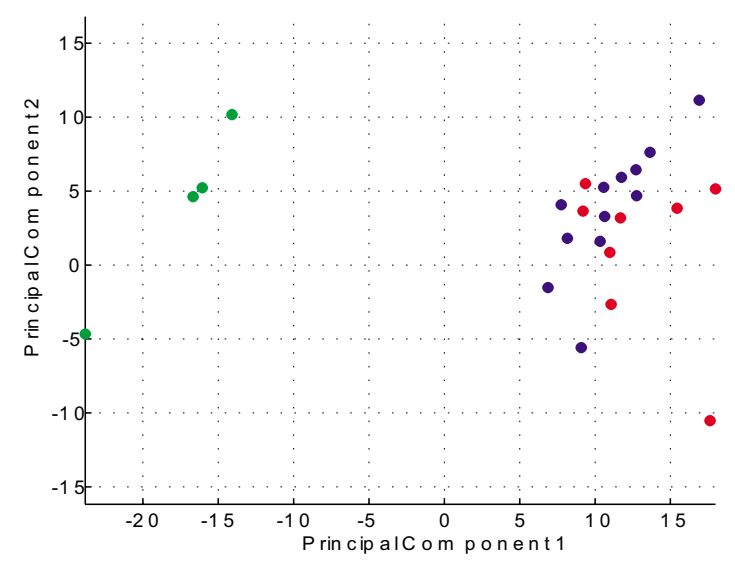

Figure 3

Principal component of current, former and never smokers using $(A)$ the 161 tags deemed reversible upon smoking cessation (Additional file 6) and (B) the 152 tags deemed irreversible upon smoking cessation (Additional file 7).

Expression values used were scaled to tags per million (TPM). Each tag was then normalized by dividing its value by the maximum value for that tag seen in all the libraries. Subsequently, this value was then multiplied by 6 and then subtracted by 3 to put the values in the range of -3 to 3 . A covariance based approach was used and the statistics toolbox in MatLab (Mathworks) was used. Current smokers are represented in red, former smokers are represented in blue and never smokers are represented in green.
A similar approach to that described here was undertaken by Spira et al. where the expression of 13 genes, including some putative oncogenes and tumor suppressor genes, was deemed irreversible upon cessation of smoking. However, none of these 13 genes overlapped with those identified in our study. This lack of overlap may reflect the differing locations from which the bronchial brushings were obtained as Spira et al [5] sampled from the right main bronchus whereas we have sampled peripheral subsegmental airways.

It is interesting to note that MUC5AC appears in both the lists of statistically reversible and irreversible gene expression changes suggesting that expression of this gene exhibits distinct states of expression among current, former and never smokers. Moreover, it should also be noted that although 311 of the 609 tags were classified as either reversible or irreversible, the remaining 298 tags did not meet the statistical criteria for either category.

\section{Validation of select gene expression changes using quantitative RT-PCR}

In addition to the SAGE analysis, which identified genes associated with airway mucosal response and xenobiotic/ nucleic acid metabolism as distinguishing features between current and former smokers, we have performed quantitative RT-PCR on a secondary cohort of current, former and never smokers to validate selected genes for expression changes (Additional file 9). In total, five genes were selected for validation. From the set of reversible genes, we have chosen CABYR, ENTPD8, and TFF3 because their expression has not been associated with smoking previously. In addition, from the irreversible genes, we have selected MUC5AC. Using the delta-delta$\mathrm{Ct}$ method to derive expression values, we then employed a Mann Whitney U Test to determine significance. The pattern of reversible over-expression in current smokers for CABYR, ENTPD8, and TFF3 (Figure 4A) and the irreversible over-expression of MUC5AC (Figure 4B) observed from the SAGE data, was validated by quantitative RT-PCR (Additional file 10). Raw cycle thresholds for each gene are available in Additional file 9.

\section{Airway epithelium response genes and their role in inflammation and cancer}

Although the role of xenobiotic metabolism in smokinginduced carcinogenesis has been well documented $[9,15]$, the potential influence mediated by changes in the composition of the airway mucosa in the development of lung cancer, has not been thoroughly investigated. It is possible that constant dysregulation of expression of genes associated with mucus secretion (such as TFF3 and MUC5AC) by smoking could potentially have a direct or indirect role in smoking-induced carcinogenesis. 
Table 3: Reversible gene expression upon smoking cessation related to mucus secretion (genes in bold have not been previously associated with smoking)

\begin{tabular}{|c|c|c|c|c|c|c|}
\hline Tag & Gene Symbol & Gene Name & CS Mean* & FS Mean* & NS Mean* & P-Value (CVsF) \\
\hline GAATGAACTG & EDIL3 & EGF-like repeats and discoidin I-like domains 3 & 72 & 5 & 8 & 0.00011 \\
\hline CTCCACCCGA & TFF3 & Trefoil factor 3 (intestinal) & 4974 & 1978 & 1722 & 0.00019 \\
\hline GTGGAGAAGA & CLDNIO & Claudin 10 & 89 & 23 & 26 & 0.00019 \\
\hline GGAATTGCCC & BPILI & Bactericidal/permeability-increasing protein-like I & 43 & 4 & 4 & 0.00029 \\
\hline TTGGTTTTTG & CXCL6 & Chemokine (C-X-C motif) ligand 6 & 147 & 414 & 371 & 0.0003 \\
\hline CCTATCAGTA & MSMB & Microseminoprotein, beta- & $1588 \mid$ & 4405 & 2948 & 0.00048 \\
\hline СTTCCTGTGA & SBEM & Small breast epithelial mucin & 154 & 27 & 32 & 0.00071 \\
\hline TGGAAATGTG & CBARAI & Calcium binding atopy-related autoantigen I & 49 & 18 & 15 & 0.00141 \\
\hline CAAGCATAAA & CABYR & Calcium binding tyrosine-(Y)-phosphorylation regulated & 63 & 4 & 4 & $0.0024 I$ \\
\hline AGGGAGGCAG & SCGBIAI & Secretoglobin, family IA, member I & 135 & 473 & 436 & 0.0041 \\
\hline TATCACATTC & CXCL6 & Chemokine (C-X-C motif) ligand 6 & 10 & 42 & 29 & 0.00573 \\
\hline TTGCACCCTT & MSMB & Microseminoprotein, beta- & 71 & 16 & 9 & 0.00735 \\
\hline AGCTTAATGA** & SCGBIAI & Secretoglobin, family IA, member I & 557 & 1478 & 3269 & 0.00956 \\
\hline GAAAAAATAG & SCGBIAI & Secretoglobin, family IA, member I (uteroglobin) & 88 & 288 & 281 & 0.0124 \\
\hline GTGGCCACGG & SIO0A9 & SI 00 calcium binding protein A9 (calgranulin B) & 26 & 101 & 63 & 0.0124 \\
\hline AAAATGTATT & CAV2 & Caveolin 2 & 20 & 6 & 4 & 0.0144 \\
\hline GACAAGGATG & CX3CLI & Chemokine ( $\mathrm{C}-\mathrm{X} 3-\mathrm{C}$ motif) ligand I & 29 & 63 & 93 & 0.01586 \\
\hline
\end{tabular}

*Mean in tags per million (TPM)

** Changed mapping with TAGMapper [56]

One of the many genes involved in lung cancer development is cyclooxygenase 2 (COX2), which plays a multi-faceted role in cellular proliferation, migration and invasiveness [41]. Notably, secretoglobin, family 1A, member 1 (SCGB1A1) protein has been shown to inhibit COX2 at the mRNA level $[42,43]$. We observed that SCGB1A1 expression is drastically reduced in current smokers but is expressed at similar levels in former and never smokers, and a previous study showed decreased serum SCGB1A1 level in smokers [44]. It should also be noted that none of the SAGE sequence tags identified in the analysis mapping to SCGB1A1 are the most reliable tag according to SAGE Genie [45]. However, even though the most reliable tag to this gene, CTTTGAGTCC did not pass statistically, the trend of reduced expression in current smokers relative to former smokers and similar expression between former and never smokers is consistent with the sequence tags that did appear in the analysis. Moreover, given that multiple tags have appeared from our analysis, although not as reliably mapped, we are confident that we are detecting SCGB1A1 mRNA expression. Interestingly, COX2 mRNA expression was not detected in the bronchial epithelium of current, former and never smokers from our SAGE data. A recent report demonstrated a significant increase in COX2 expression in normal lung fibroblasts when exposed to cigarette smoke extracts [46]. It is possible that SCGB1A1 involvement is in the stroma and not in epithelial cells.

Despite lack of knowledge about $C A B Y R$, one of its few known interactions occurs with GSK3B [30].CABYR is a substrate of GSK3B [30], and exhibits reversible, increased expression with active smoking (Figure 4A). Though GSK3B was not identified as a smoking-related gene in our primary analysis, investigation of the SAGE data revealed a trend of similar decreased expression in current and former smokers relative to never smokers. Moreover, quantitative RT-PCR using a secondary cohort of samples validated that GSK3B expression is irreversibly reduced in ever smokers (Figure 4B). Recently, a published report using porcine tracheobronchial epithelial cells exposed to cigarette smoke components in vitro, demonstrated an inhibition of GSK3B gene expression [47]. GSK3B has been shown to negatively interact with COX2 [48]. Reduced expression of GSK3B may therefore account for exaggerated inflammatory response despite smoking cessation and may contribute to development of lung cancer.

In this study, we have demonstrated differential expression of various components of respiratory tract mucus (including TFF3 and MUC5AC) according to smoking status (Table 3). However, our data indicates that MUC5AC expression is not completely reversible upon smoking cessation and in fact, exhibits three statistically distinct levels of expression between current, former and never smokers (Figure 4B). TFF2, a related motogen to TFF3, in conjunction with epidermal growth factor (EGF), has been shown to promote airway restitution, (i.e., movement of neighboring airway epithelial cells in response to injury mimicking rapid epithelium regeneration), through the activation of the epidermal growth factor receptor (EGFR) [49], expressed in the normal bronchial mucosa [50,51]. Other studies have also demonstrated increased expression of MUC5AC, along with EGFR and v-erb-b2 erythroblastic leukemia viral oncogene homolog 3 (ERBB3) in active smokers $[26,32]$. We examined EGFR expression in relation to smoking and found that there was a modest increase of approximately 1.5-fold between current and former smokers in our SAGE data. As enhanced expression of EGFR is well documented in lung cancer $[52,53]$, these 
A
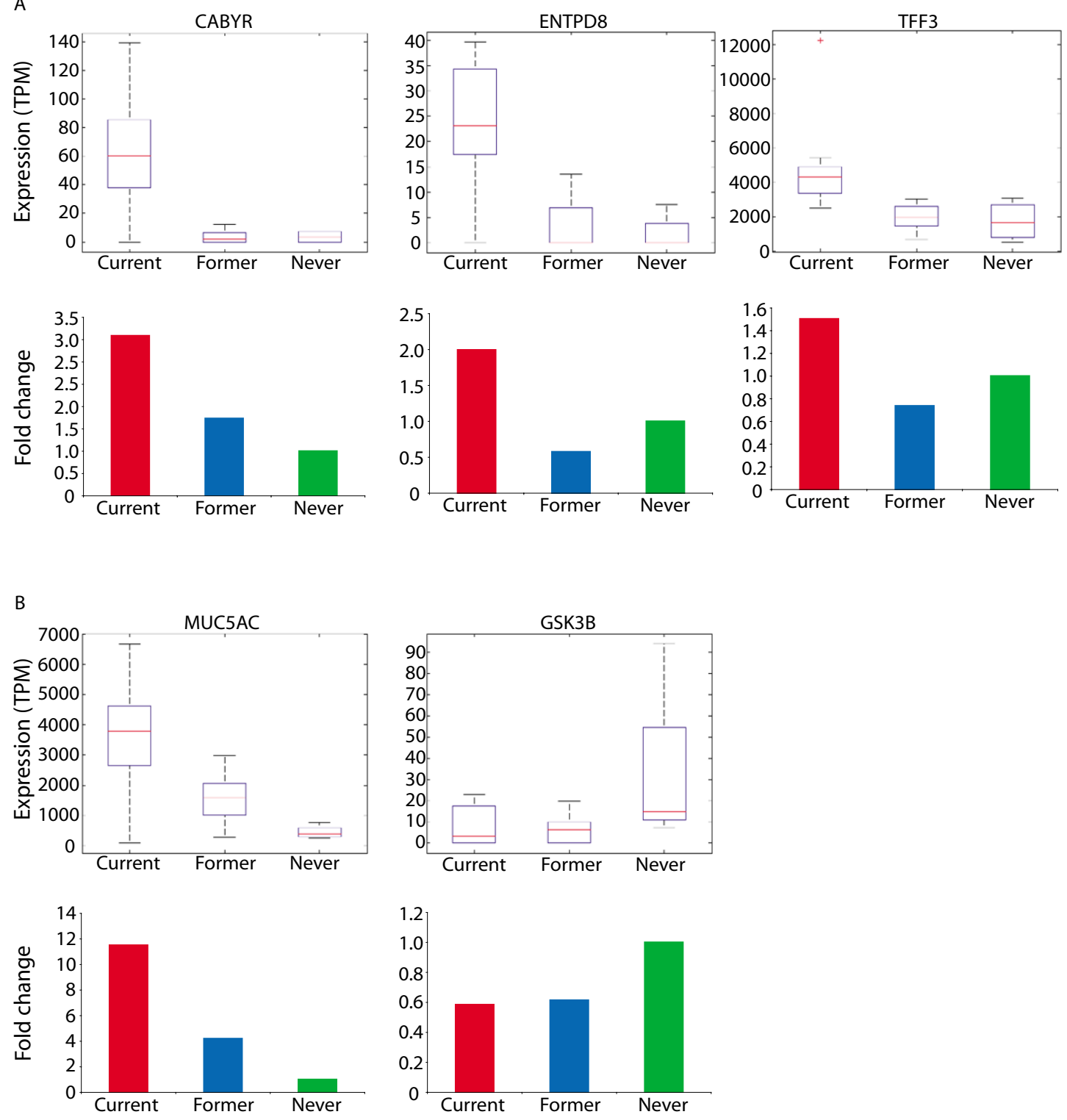

\section{Figure 4}

SAGE and quantitative PCR (qRT-PCR) analysis of select genes: (A) Genes found to have reversible expression upon smoking cessation. Box plots of SAGE data and histograms for qRT-PCR for CABYR, ENTPD8 and TFF3. Distribution of ratios between both current vs. former and current vs. former and never (Additional file IV) were found to be statistically different. (B) Genes found to be either partially or fully irreversible. Box plots of SAGE data and histograms for qRT-PCR for MUC5AC and GSK3B. Distribution of ratios between current vs. former and former vs. never were statistically different for MUC5AC and in addition, GSK3B was statistically significant for the combination of current and former vs. never. Box plot analysis was done using the Statistics toolbox from the MathWorks MatLab program. Red lines in the boxes represent the median expression value in terms of tags per million (TPM), and red "plus" signs represent outliers (values which are greater than I.5 times the maximum value). The bottom and top part of the boxes represent the $2^{\text {nd }}$ and $3^{\text {rd }}$ quartiles of the data respectively. The error bars represent the $5^{\text {th }}$ and $95^{\text {th }}$ percentiles of the data. Quantitative RT-PCR validation was performed on a second cohort of nine current smokers, seven former smokers and six never smokers. Plotted is the average expression ratio relative to the average expression in never smokers of current (red), former (blue) and never (green) smokers. Statistical significance was determined using a onetailed p-value from the Mann Whitney U Test (Supplemental Table IX). 
results imply that enhanced expression of TFF3 (and perhaps other genes associated with airway epithelial response and mucus secretion) may promote airway restitution in response to active smoking and that constant induction of airway reconstruction may play a role in the development of lung cancer (Figure 5).

\section{Conclusion}

This study represents the largest human SAGE study reported to date. Over three million SAGE tags were sequenced, representing over 110 thousand potentially unique transcripts expressed within the bronchial epithelium relative to cigarette smoke exposure. These libraries provide a valuable resource for future data mining. Based on the gene expression profiles of 24 current, former and never smokers, we identified both reversible and irreversible gene expression changes upon smoking cessation. Specifically, amongst those genes reversibly expressed, three main functions were identified: xenobiotic metabo-

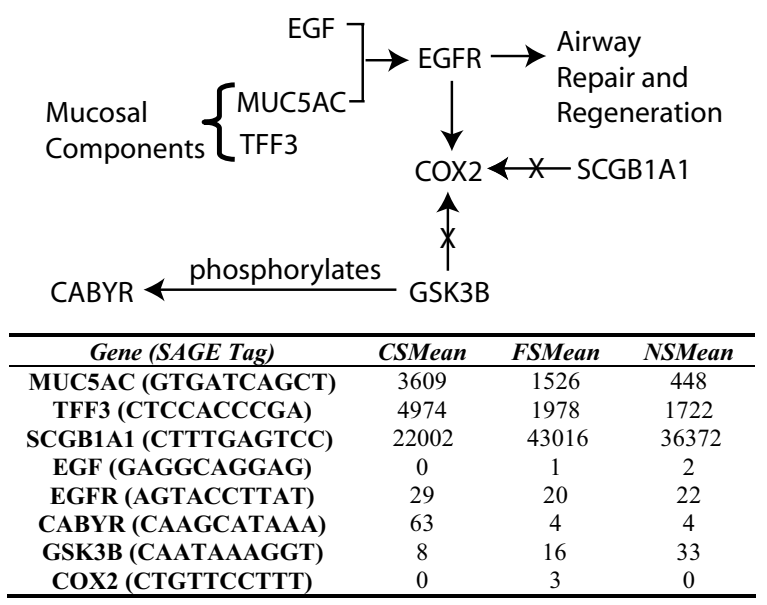

\section{Figure 5}

Expression trends of specific genes related to muco-ciliary function and airway restitution as compared with smoking status and lung cancer: TFF3, CABYR, and MUC5AC are over expressed in current smokers with lowered expression in both former and never smokers. Conversely, SCGBIAI shows the opposite effect, with lowered expression in current smokers as compared to former and never smokers. MUC5AC and TFF3 are known to be components of mucus. EGFR levels are positively correlated with smoking status, with modestly higher levels in current smokers. MUC5AC and EGF have been shown to interact with EGFR in the process of airway restitution and SCGBIAI has been shown to decrease levels of cyclooxygenase 2 (COX2) in cancer cells. Interestingly, within this process alone, we see reversible (TFF3, CABYR), partially reversible (MUC5AC) and completely irreversible (GSK3B) expression changes upon smoking cessation. Values refer to tag counts as tags-per-million (TPM). lism, nucleotide metabolism, and mucus secretion. In addition, some of the genes associated with airway mucosal response are strongly involved with airway epithelium repair and regeneration. Interestingly, investigating airway repair and regeneration revealed genes varying in the degree of reversibility, including those completely reversible (TFF3, CABYR), partially reversible (MUC5AC) and irreversible (GSK3B) expression changes upon smoking cessation. We have validated the SAGE expression data for TFF3, CABYR, MUC5AC, GSK3B and ENTPD8 using a secondary cohort of current, former and never smokers. This is the first study demonstrating smoking-induced expression changes for this particular set of genes and importantly, it is the first time partial reversibility (MUC5AC) and irreversibility (GSK3B) and has been demonstrated using two different cohorts of samples with two independent assays for expression quantification. By comprehensively identifying gene expression changes that are reversible upon smoking cessation, we have introduced genes which may in future studies be investigated for polymorphisms, as those genes which are not sufficiently induced in response to smoking may identify candidate loci of susceptibility. Similarly, those genes and functions which do not revert to normal levels upon smoking cessation may also provide insight into why former smokers still maintain a risk of developing lung cancer.

\section{Methods \\ Specimen collection}

Bronchial epithelial cells were collected by bronchial brushings from 24 subjects - 9 current smokers, 11 former smokers and 4 never smokers summarized in Table 1 - by bronchial brushing as described previously $[54,55]$. The subjects were volunteer smokers recruited from the community as part of a NCI-sponsored chemoprevention trial. The inclusion criteria were: age $>45$ years of age and a smoking history of $\geq 30$ pack years. A former smoker was defined as one who had stopped smoking for at least one year or more. None of the subjects were on bronchodilator or inhaled steroids. The samples were obtained prior to treatment with an investigational chemoprevention agent.

Brushings were obtained from the peripheral airways using a $1.8 \mathrm{~mm}$ brush. A table of the basic demographics of the subjects used is listed in Table 1.

\section{Construction of SAGE libraries}

To deduce the gene expression profiles, we used a method called serial analysis of gene expression (SAGE) which quantifies gene expression by the enumeration of transcript derived sequence tags [20]. SAGE libraries were constructed from each sample using the MicroSAGE protocol [55], and sequenced to a depth of $~ 150,000$ SAGE tags per 
library. SAGE libraries were deposited in NCBI GEO with accession number GSE5473. Reproducibility of SAGE libraries obtained from the same bronchial brush was shown by our group previously. The $\mathrm{R}$ value between two libraries from the same lysate was 0.97 [55].

\section{SAGE tag-to-gene mapping}

Tag-to-gene mapping was performed using a combination of the May $10^{\text {th }}, 2006$ build of SAGEGenie [45]. Tags with low reliability from SAGEGenie in Table 2 and 3 were also cross-referenced with TagMapper [56].

\section{Statistical analysis of differentially expressed genes}

Stringently, only tags which exhibited a mean tag count of $\geq 20$ tags per million (TPM) in at least one of current, former or never smoker SAGE libraries were used in comparative analysis. For each specific comparison, in addition to the tag count requirement, a minimum fold change of the means of two was also required. The tag abundance requirement of a mean tag count of 20 TPM was used to filter the list of tags prior to statistical comparison to reduce the number of false positives. 8148 tags meet this criterion. Given the variability in smokers and limited sample size in this study, a non-parametric Mann Whitney U Test was used to determine if a given tag (representing a gene) was differentially expressed using a pvalue threshold of $\mathrm{p} \leq 0.05$, unadjusted for multiple comparisons.

\section{Validation of SAGE-specific targets using quantitative RT- PCR}

Select targets identified in the SAGE study were validated using quantitative RT-PCR (qRT-PCR) in a second cohort of nine current, seven former and six never smokers. Briefly, $100 \mathrm{ng}$ of RNA was isolated and converted to cDNA in a $50 \mu \mathrm{l}$ reaction volume using the High-Capacity cDNA Archive Kit (cat \# 4322171, Applied Biosystems). 1 $\mu \mathrm{l}$ of the resulting cDNA was analysed by qPCR, with specified Taqman primers and TaqMan Universal PCR Master Mix (cat \# 4326708), using the iCycler iQTM Real-Time PCR Detection System (Bio-Rad). CABYR, TFF3, MUC5AC, GSK3B and Actin Beta were monitored for 40 cycles of PCR and ENTPD8 for 50 cycles. Primers used for qRT-PCR are listed in Additional file 11.

\section{Authors' contributions}

RC analyzed the SAGE and quantitative PCR data to deduce the key findings, and wrote the manuscript.

KML led the construction of all SAGE libraries and contributed to data interpretation and manuscript editing.

RTN provided insight to statistical analysis.
CM provided insight to statistical analysis as well as manuscript editing.

SL isolated the clinical samples from current, former and never smokers, and contributed to interpretation of results.

SL and WLL are the principal investigators of this project.

\section{Additional material}

\section{Additional file 1}

Supplementary Table 1 - Tags expressed in all current smoker libraries. Tags which have a raw count of greater than 2 in all 8 current smoker SAGE libraries.

Click here for file

[http://www.biomedcentral.com/content/supplementary/1471-

2164-8-297-S1.xls]

\section{Additional file 2}

Supplementary Table 2 - Tags expressed in all former smoker libraries. Tags which have a raw count of greater than 2 in all 12 former smoker SAGE libraries.

Click here for file

[http://www.biomedcentral.com/content/supplementary/14712164-8-297-S2.xls]

\section{Additional file 3}

Supplementary Table 3 - Tags expressed in all never smoker libraries. Tags which have a raw count greater than 2 in all 4 never smoker SAGE libraries.

Click here for file

[http://www.biomedcentral.com/content/supplementary/14712164-8-297-S3.xls]

\section{Additional file 4}

Supplementary Table 4 - Tags expressed in all 24 SAGE libraries. Tags which have a raw count greater than 2 in all 24 SAGE libraries.

Click here for file

[http://www.biomedcentral.com/content/supplementary/1471-

2164-8-297-S4.xls]

\section{Additional file 5}

Supplementary Table 5 - 609 tags differentially expressed between current and never smokers. 609 differentially expressed tags between current and never smokers.

Click here for file

[http://www.biomedcentral.com/content/supplementary/14712164-8-297-S5.xls]

\section{Additional file 6}

Supplementary Table $6-161$ tags with reversible expression upon smoking cessation. 161 tags which exhibit statistically reversible gene expression upon smoking cessation.

Click here for file

[http://www.biomedcentral.com/content/supplementary/14712164-8-297-S6.xls] 


\section{Additional file 7 \\ Supplementary Table 7 - 152 tags with irreversible expression upon smok- ing cessation. 152 tags which exhibit statistically irreversible gene expres- sion upon smoking cessation. \\ Click here for file \\ [http://www.biomedcentral.com/content/supplementary/1471- \\ 2164-8-297-S7.xls] \\ Additional file 8 \\ Supplementary Table 8 - 157 tags differentially expressed between mild and moderate/severe COPD. \\ Click here for file \\ [http://www.biomedcentral.com/content/supplementary/1471- \\ 2164-8-297-S8.xls \\ Additional file 9 \\ Supplementary Table 9-Cycle threshold data from quantitative RT-PCR. Raw cycle threshold data for quantitative RT-PCR of 5 genes. \\ Click here for file \\ [http://www.biomedcentral.com/content/supplementary/1471- \\ 2164-8-297-S9.xls] \\ Additional file 10 \\ Supplementary Table 10 - Fold-changes and p-values from quantitative RT-PCR analysis. Data from the analysis of the quantitative RT-PCR results. \\ Click here for file \\ [http://www.biomedcentral.com/content/supplementary/1471- 2164-8-297-S10.xls] \\ Additional file 11 \\ Supplementary Table 11 - Quantitative RT-PCR primers. Primers ordered from Applied Biosystems for 5 genes. \\ Click here for file \\ [http://www.biomedcentral.com/content/supplementary/1471- 2164-8-297-S11.xls]}

\section{Acknowledgements}

We thank William W. Lockwood, Jonathan J. Davies, Bradley P. Coe, lan M. Wilson and Teresa L. Mastracci for useful discussion. We also would like to thank Andrea Pusic for assistance with quantitative RT-PCR validation and SAGE library construction and Baljit Kamoh and Blair Gervan for assistance with SAGE library construction. This work was supported by funds from Genome Canada/Genome British Columbia, Canadian Institutes of Health Research, and NIDCR grant ROI DEI5965-0I. RC is supported by scholarships from the Canadian Institutes of Health Research and the Michael Smith Foundation for Health Research.

\section{References}

I. Jemal A, Siegel R, Ward E, Murray T, Xu J, Smigal C, Thun MJ: Cancer statistics, 2006. CA Cancer J Clin 2006, 56(2): I06-I30.

2. Halpern MT, Gillespie BW, Warner KE: Patterns of absolute risk of lung cancer mortality in former smokers. J Natl Cancer Inst 1993, 85(6):457-464.

3. Peto R, Darby S, Deo H, Silcocks P, Whitley E, Doll R: Smoking, smoking cessation, and lung cancer in the UK since 1950: combination of national statistics with two case-control studies. Bmj 2000, 32 I(7257):323-329.

4. Tong L, Spitz MR, Fueger JJ, Amos CA: Lung carcinoma in former smokers. Cancer 1996, 78(5): 1004-1010.
5. Spira A, Beane J, Shah V, Liu G, Schembri F, Yang X, Palma J, Brody JS: Effects of cigarette smoke on the human airway epithelial cell transcriptome. Proc Natl Acad Sci U S A 2004, I0 I(27): $10 \mid 43-10148$.

6. Sutter TR, Tang YM, Hayes CL, Wo YY, Jabs EW, Li X, Yin H, Cody $C W$, Greenlee WF: Complete cDNA sequence of a human dioxin-inducible mRNA identifies a new gene subfamily of cytochrome P450 that maps to chromosome 2. J Biol Chem 1994, 269( I 8): I 3092-I 3099.

7. Shimada T, Hayes CL, Yamazaki H, Amin S, Hecht SS, Guengerich FP, Sutter TR: Activation of chemically diverse procarcinogens by human cytochrome P-450 IBI. Cancer Res 1996, 56(I 3):2979-2984.

8. Kim JH, Stansbury KH, Walker NJ, Trush MA, Strickland PT, Sutter TR: Metabolism of benzo[a]pyrene and benzo[a]pyrene-7,8diol by human cytochrome P450 IBI. Carcinogenesis 1998, I9(I0): I847-1853.

9. Fukumoto S, Yamauchi N, Moriguchi H, Hippo Y, Watanabe A, Shibahara J, Taniguchi $H$, Ishikawa S, Ito $H$, Yamamoto S, Iwanari H, Hironaka $M$, Ishikawa $Y$, Niki T, Sohara $Y$, Kodama T, Nishimura $M$, Fukayama M, Dosaka-Akita H, Aburatani H: Overexpression of the aldo-keto reductase family protein AKR IB I 0 is highly correlated with smokers' non-small cell lung carcinomas. Clin Cancer Res 2005, I I(5): I 776-I785.

10. Piipari R, Nurminen T, Savela K, Hirvonen A, Mantyla T, Anttila S: Glutathione S-transferases and aromatic DNA adducts in smokers' bronchoalveolar macrophages. Lung Cancer 2003, 39(3):265-272.

II. Piipari R, Savela K, Nurminen T, Hukkanen J, Raunio H, Hakkola J, Mantyla T, Beaune P, Edwards RJ, Boobis AR, Anttila S: Expression of CYPIAI, CYPIBI and CYP3A, and polycyclic aromatic hydrocarbon-DNA adduct formation in bronchoalveolar macrophages of smokers and non-smokers. Int J Cancer 2000, 86(5):610-616.

12. Benowitz NL, Jacob $P$ 3rd: Nicotine and cotinine elimination pharmacokinetics in smokers and nonsmokers. Clin Pharmacol Ther 1993, 53(3):316-323.

13. Phillips DH, Schoket B, Hewer A, Bailey E, Kostic S, Vincze I: Influence of cigarette smoking on the levels of DNA adducts in human bronchial epithelium and white blood cells. Int / Cancer 1990, 46(4):569-575

14. Vineis $P$, Veglia F, Anttila S, Benhamou S, Clapper ML, Dolzan V Ryberg D, Hirvonen A, Kremers P, Le Marchand L, Pastorelli R, Rannug A, Romkes M, Schoket B, Strange RC, Garte S, Taioli E: CYPIAI, GSTMI and GSTTI polymorphisms and lung cancer: a pooled analysis of gene-gene interactions. Biomarkers 2004, 9(3):298-305.

15. Larsen JE, Colosimo ML, Yang IA, Bowman R, Zimmerman PV, Fong KM: Risk of non-small cell lung cancer and the cytochrome P450I Al Ile462Val polymorphism. Cancer Causes Control 2005, I 6(5):579-585.

16. Coles SJ, Levine LR, Reid L: Hypersecretion of mucus glycoproteins in rat airways induced by tobacco smoke. Am J Pathol 1979, 94(3):459-47|.

17. Lamb D, Reid L: Goblet cell increase in rat bronchial epithelium after exposure to cigarette and cigar tobacco smoke. $\mathrm{Br}$ Med J 1969, I(5635):33-35.

18. Hovenberg HW, Davies JR, Herrmann A, Linden CJ, Carlstedt I: MUC5AC, but not MUC2, is a prominent mucin in respiratory secretions. Glycoconj J 1996, I 3(5):839-847.

19. Takeyama K, Jung B, Shim J], Burgel PR, Dao-Pick T, Ueki IF, Protin U, Kroschel P, Nadel JA: Activation of epidermal growth factor receptors is responsible for mucin synthesis induced by cigarette smoke. Am J Physiol Lung Cell Mol Physiol 200I, 280(I):LI65-72.

20. Velculescu VE, Zhang L, Vogelstein B, Kinzler KW: Serial analysis of gene expression. Science 1995, 270(5235):484-487.

21. Weeraratna AT, Becker D, Carr KM, Duray PH, Rosenblatt KP, Yang S, Chen Y, Bittner M, Strausberg RL, Riggins G], Wagner U, Kallioniemi OP, Trent JM, Morin PJ, Meltzer PS: Generation and analysis of melanoma SAGE libraries: SAGE advice on the melanoma transcriptome. Oncogene 2004, 23(I 2):2264-2274.

22. Perez-Plasencia C, Riggins G, Vazquez-Ortiz G, Moreno J, Arreola $H$, Hidalgo A, Pina-Sanchez P, Salcedo M: Characterization of the global profile of genes expressed in cervical epithelium by 
Serial Analysis of Gene Expression (SAGE). BMC Genomics 2005, 6:130.

23. Sturn A, Quackenbush J, Trajanoski Z: Genesis: cluster analysis of microarray data. Bioinformatics 2002, I8(I):207-208.

24. Pierrou S, Broberg P, O'Donnell R A, Pawlowski K, Virtala R, Lindqvist E, Richter A, Wilson SJ, Angco G, Moller S, Bergstrand H, Koopmann W, Wieslander E, Stromstedt PE, Holgate ST, Davies DE, Lund J, Djukanovic R: Expression of genes involved in oxidative stress responses in airway epithelial cells of smokers with chronic obstructive pulmonary disease. Am J Respir Crit Care Med 2007, 175(6):577-586.

25. Woenckhaus M, Klein-Hitpass L, Grepmeier U, Merk J, Pfeifer M, Wild P, Bettstetter M, Wuensch P, Blaszyk H, Hartmann A, Hofstaedter F, Dietmaier W: Smoking and cancer-related gene expression in bronchial epithelium and non-small-cell lung cancers. J Pathol 2006, 21 0(2): 192-204.

26. Wiede A, Jagla W, Welte T, Kohnlein T, Busk H, Hoffmann W: Localization of TFF3, a new mucus-associated peptide of the human respiratory tract. Am J Respir Crit Care Med 1999, I 59(4 Pt I): I330-1335.

27. Graness A, Chwieralski CE, Reinhold D, Thim L, Hoffmann W: Protein kinase $C$ and ERK activation are required for TFF-peptide-stimulated bronchial epithelial cell migration and tumor necrosis factor-alpha-induced interleukin-6 (IL-6) and IL-8 secretion. J Biol Chem 2002, 277(2 I): I8440-I8446.

28. Naaby-Hansen S, Mandal A, Wolkowicz MJ, Sen B, Westbrook VA, Shetty J, Coonrod SA, Klotz KL, Kim YH, Bush LA, Flickinger CJ, Herr JC: CABYR, a novel calcium-binding tyrosine phosphorylation-regulated fibrous sheath protein involved in capacitation. Dev Biol 2002, 242(2):236-254.

29. Eliakim R, Fan QX, Babyatsky MW: Chronic nicotine administration differentially alters jejunal and colonic inflammation in interleukin- 10 deficient mice. Eur J Gastroenterol Hepatol 2002, |4(6):607-6|4.

30. Hsu HC, Lee YL, Cheng TS, Howng SL, Chang LK, Lu PJ, Hong YR: Characterization of two non-testis-specific CABYR variants that bind to GSK3beta with a proline-rich extensin-like domain. Biochem Biophys Res Commun 2005, 329(3): I 108-III 7.

31. Weiber H, Andersson C, Murne A, Rannevik G, Lindstrom C, Lilja H, Fernlund P: Beta microseminoprotein is not a prostate-specific protein. Its identification in mucous glands and secretions. Am J Pathol 1990, I37(3):593-603.

32. O'Donnell RA, Richter A, Ward J, Angco G, Mehta A, Rousseau K, Swallow DM, Holgate ST, Djukanovic R, Davies DE, Wilson SJ: Expression of ErbB receptors and mucins in the airways of long term current smokers. Thorax 2004, 59( I 2): I032-1040.

33. Ning W, Li CJ, Kaminski N, Feghali-Bostwick CA, Alber SM, Di YP, Otterbein SL, Song R, Hayashi S, Zhou Z, Pinsky DJ, Watkins SC, Pilewski JM, Sciurba FC, Peters DG, Hogg JC, Choi AM: Comprehensive gene expression profiles reveal pathways related to the pathogenesis of chronic obstructive pulmonary disease. Proc Natl Acad Sci U S A 2004, I0 I (4 I): I 4895-14900.

34. Ito H, Matsuo K, Hamajima N, Mitsudomi T, Sugiura T, Saito T, Yasue T, Lee KM, Kang D, Yoo KY, Sato S, Ueda R, Tajima K: Gene-environment interactions between the smoking habit and polymorphisms in the DNA repair genes, APEI Asp I48Glu and XRCCI Arg399GIn, in Japanese lung cancer risk. Carcinogenesis 2004, 25(8): I 395-I40I.

35. Yuasa MS, Masutani C, Hirano A, Cohn MA, Yamaizumi M, Nakatan Y, Hanaoka F: A human DNA polymerase eta complex containing Rad 18, Rad6 and RevI; proteomic analysis and targeting of the complex to the chromatin-bound fraction of cells undergoing replication fork arrest. Genes Cells 2006, II(7):73I-744.

36. Tissier A, Kannouche P, Reck MP, Lehmann AR, Fuchs RP, Cordonnier $A$ : Co-localization in replication foci and interaction of human Y-family members, DNA polymerase pol eta and REVI protein. DNA Repair (Amst) 2004, 3(I I): I503-I5I4.

37. Ueda K, Kawashima H, Ohtani S, Deng WG, Ravoori M, Bankson J Gao B, Girard L, Minna JD, Roth JA, Kundra V, Ji L: The 3p2I.3 Tumor Suppressor NPRL2 Plays an Important Role in Cisplatin-Induced Resistance in Human Non-Small-Cell Lung Cancer Cells. Cancer Res 2006, 66(19):9682-9690.

38. Zamble DB, Lippard SJ: Cisplatin and DNA repair in cancer chemotherapy. Trends Biochem Sci 1995, 20( I 0):435-439.
39. Pauwels RA, Buist AS, Calverley PM, Jenkins CR, Hurd SS: Global strategy for the diagnosis, management, and prevention of chronic obstructive pulmonary disease. NHLBI/WHO Global Initiative for Chronic Obstructive Lung Disease (GOLD) Workshop summary. Am J Respir Crit Care Med 2001, 163(5): 1256-1276.

40. Gross N]: The GOLD standard for chronic obstructive pulmonary disease. Am J Respir Crit Care Med 200I, I 63(5): 1047-1048.

4l. Sheng H, Shao J, Washington MK, DuBois RN: Prostaglandin E2 increases growth and motility of colorectal carcinoma cells. J Biol Chem 200I, 276(2I): I8075-1808I.

42. Dierynck I, Bernard A, Roels H, De Ley M: Potent inhibition of both human interferon-gamma production and biologic activity by the Clara cell protein CCI6. Am J Respir Cell Mol Biol I995, I 2(2):205-210.

43. Hermans C, Bernard A: Lung epithelium-specific proteins: characteristics and potential applications as markers. Am J Respir Crit Care Med 1999, 159(2):646-678.

44. Robin M, Dong P, Hermans C, Bernard A, Bersten AD, Doyle IR: Serum levels of CCI 6, SP-A and SP-B reflect tobacco-smoke exposure in asymptomatic subjects. Eur Respir J 2002, 20(5): || $|52-||6|$

45. Boon K Osorio EC, Greenhut SF, Schaefer CF, Shoemaker J, Polyak K, Morin PJ, Buetow KH, Strausberg RL, De Souza SJ, Riggins GJ: An anatomy of normal and malignant gene expression. Proc Nat Acad Sci U S A 2002, 99(I7): | | 287- I | 292

46. Martey CA, Pollock SJ, Turner CK, O'Reilly KM, Baglole CJ, Phipps RP, Sime PJ: Cigarette smoke induces cyclooxygenase-2 and microsomal prostaglandin E2 synthase in human lung fibroblasts: implications for lung inflammation and cancer. $\mathrm{Am} J$ Physiol Lung Cell Mol Physiol 2004, 287(5):L98I-9I.

47. Tian D, Zhu M, Chen WS, Li JS, Wu RL, Wang X: Role of glycogen synthase kinase 3 in squamous differentiation induced by cigarette smoke in porcine tracheobronchial epithelial cells. Food Chem Toxicol 2006, 44(9): I590-I596.

48. Thiel A, Heinonen M, Rintahaka J, Hallikainen T, Hemmes A, Dixon DA, Haglund C, Ristimaki A: Expression of cyclooxygenase-2 is regulated by glycogen synthase kinase-3beta in gastric cancer cells. J Biol Chem 2006, 28 I (8):4564-4569.

49. Oertel M, Graness A, Thim L, Buhling F, Kalbacher H, Hoffmann W: Trefoil factor family-peptides promote migration of human bronchial epithelial cells: synergistic effect with epidermal growth factor. Am J Respir Cell Mol Biol 200I, 25(4):418-424.

50. Barsky SH, Roth MD, Kleerup EC, Simmons M, Tashkin DP: Histopathologic and molecular alterations in bronchial epithelium in habitual smokers of marijuana, cocaine, and/or tobacco. J Natl Cancer Inst 1998, 90( I6): | | 98-1205.

5I. Yoneda K: Distribution of proliferating-cell nuclear antigen and epidermal growth factor receptor in intraepithelial squamous cell lesions of human bronchus. Mod Pathol 1994 7(4):480-486.

52. Rusch V, Baselga J, Cordon-Cardo C, Orazem J, Zaman M, Hoda S, McIntosh J, Kurie J, Dmitrovsky E: Differential expression of the epidermal growth factor receptor and its ligands in primary non-small cell lung cancers and adjacent benign lung. Cancer Res 1993, 53(10 Suppl):2379-2385.

53. Polosa R, Prosperini G, Leir SH, Holgate ST, Lackie PM, Davies DE: Expression of c-erbB receptors and ligands in human bronchial mucosa. Am J Respir Cell Mol Biol I999, 20(5):914-923.

54. Lam S, Kennedy T, Unger M, Miller YE, Gelmont D, Rusch V, Gipe B, Howard D, LeRiche JC, Coldman A, Gazdar AF: Localization of bronchial intraepithelial neoplastic lesions by fluorescence bronchoscopy. Chest 1998, I I3(3):696-702.

55. Lonergan KM, Chari R, Deleeuw RJ, Shadeo A, Chi B, Tsao MS, Jones S, Marra M, Ling V, Ng R, Macaulay C, Lam S, Lam WL: Identification of novel lung genes in bronchial epithelium by serial analysis of gene expression. Am J Respir Cell Mol Biol 2006, 35(6):65I-66I.

56. Bala P, Georgantas RW 3rd, Sudhir D, Suresh M, Shanker K, Vrushabendra BM, Civin Cl, Pandey A: TAGmapper: a web-based tool for mapping SAGE tags. Gene 2005, 364: |23-I29. 